\title{
Parabolic subgroups acting on the additional length graph
}

\author{
YAGO ANTOLÍN \\ MARÍA CUMPLIDO
}

Let $A \neq A_{1}, A_{2}, I_{2 m}$ be an irreducible Artin-Tits group of spherical type. We show that the periodic elements of $A$ and the elements preserving some parabolic subgroup of $A$ act elliptically on the additional length graph $\mathcal{C}_{\mathrm{AL}}(A)$, a hyperbolic, infinite diameter graph associated to $A$ constructed by Calvez and Wiest to show that $A / Z(A)$ is acylindrically hyperbolic. We use these results to find an element $g \in A$ such that $\langle P, g\rangle \cong P *\langle g\rangle$ for every proper standard parabolic subgroup $P$ of $A$. The length of $g$ is uniformly bounded with respect to the Garside generators, independently of $A$. This allows us to show that, in contrast with the Artin generators case, the sequence $\left\{\omega\left(A_{n}, \mathcal{S}\right)\right\}_{n \in \mathbb{N}}$ of exponential growth rates of braid groups, with respect to the Garside generating set, goes to infinity.

20F36, 20F65

\section{Introduction}

It is well known that the braid group with $n+1$ strands, $A_{n}$, acts by isometries on the curve complex of the $n$-punctured disk, $\mathcal{D}_{n}$. This fact comes from the topological definition of $A_{n}$, which says that $A_{n}$ is the mapping class group of $\mathcal{D}_{n}$. We know a lot about this curve complex, including its $\delta$-hyperbolicity, which makes it a fundamental tool when proving properties of the braid group.

On the other hand, braid groups also belong to a family of presentable groups, called Artin-Tits groups [1]. To define them we need a finite set of generators $\Sigma$ and a symmetric matrix $M=\left(m_{s, t}\right)_{s, t \in \Sigma}$ with $m_{s, s}=1$ and $m_{s, t} \in\{2, \ldots, \infty\}$ for $s \neq t$. The Artin-Tits system associated to $M$ is $(A, \Sigma)$, where $A$ is the so-called Artin-Tits group presented in the following way:

$$
A=\langle\Sigma| \underbrace{s t s \cdots}_{m_{s, t} \text { elements }}=\underbrace{t s t \cdots}_{m_{s, t} \text { elements }} \text { for } s, t \in \Sigma \text { with } s \neq t, m_{s, t} \neq \infty\rangle .
$$

Notice that relations in this presentation contain only positive powers of the generators. This allows us to define $A^{+}$as the positive monoid given by the same presentation. 

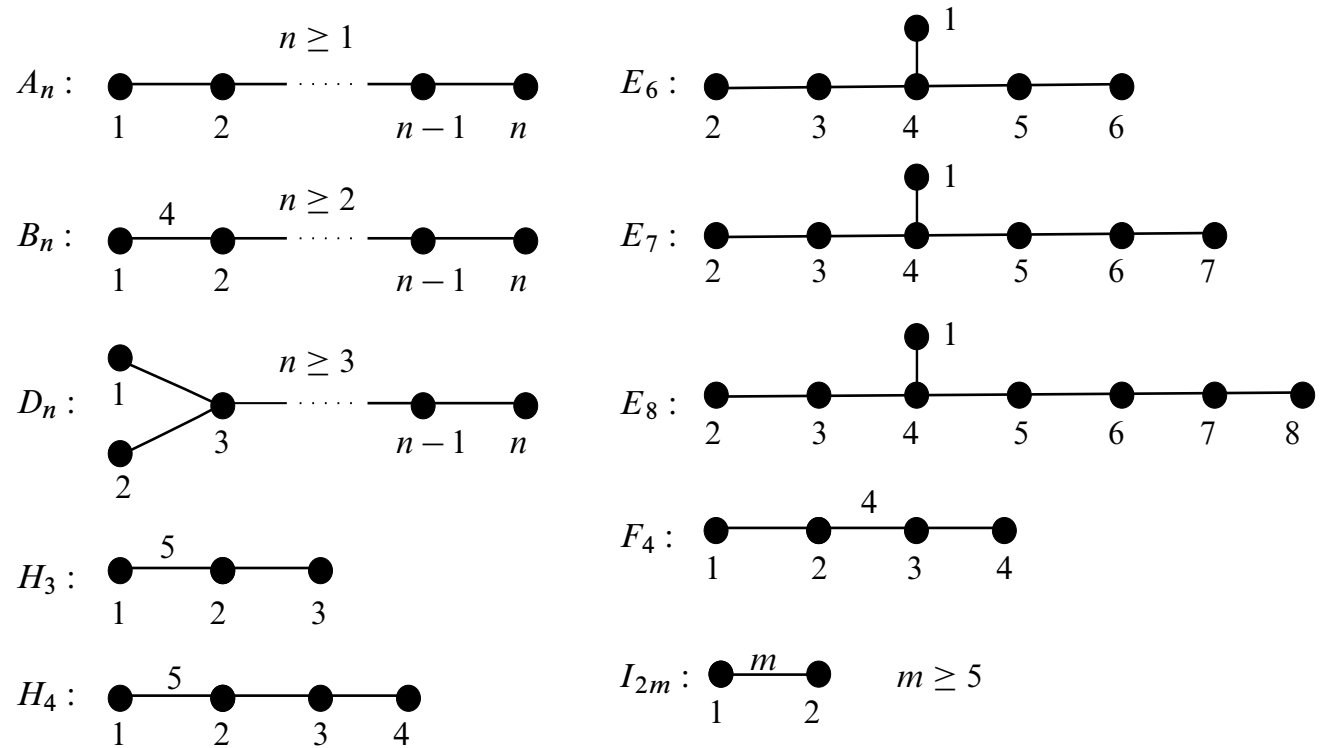

Figure 1: Classification of irreducible Coxeter graphs of finite type.

We also can obtain the Coxeter group $W_{A}$ associated to $(A, \Sigma)$ by adding the relations $s^{2}=1$ :

$W_{A}=\langle\Sigma| s^{2}=1$ for $s \in \Sigma, \underbrace{s t s \cdots}_{m_{s, t} \text { elements }}=\underbrace{t s t \cdots}_{m_{s, t} \text { elements }}$ for $s, t \in \Sigma$ with $s \neq t, m_{s, t} \neq \infty\rangle$.

If $W_{A}$ is finite, the corresponding Artin-Tits group (or Artin-Tits system) is said to be of spherical type. If $A$ cannot be decomposed as a direct product of nontrivial ArtinTits groups, we say that $A$ is irreducible. Irreducible Artin-Tits groups of spherical type are completely classified (see Figure 1) in ten classes; see Coxeter [7]. The main example on these groups is the braid group $A_{n}$, which is provided by the presentation

$$
\left.A_{n}=\left\langle\sigma_{1}, \ldots, \sigma_{n}\right| \sigma_{i} \sigma_{j}=\sigma_{j} \sigma_{i} \text { if }|i-j|>1, \sigma_{i} \sigma_{j} \sigma_{i}=\sigma_{j} \sigma_{i} \sigma_{j} \text { if }|i-j|=1\right\rangle .
$$

Artin-Tits groups of spherical type share many properties with $A_{n}$, but to prove them we cannot use arguments that involve the curve complex. To overcome this difficulty, new complexes related with all Artin-Tits groups of spherical type have been introduced.

Let $(A, \Sigma)$ be an Artin-Tits system of spherical type. On the one hand, in [5], Calvez and Wiest constructed the additional length graph of $A$, denoted by $\mathcal{C}_{\mathrm{AL}}(A)$. As we will see later, this complex relies on technical concepts about Garside theory and it is in fact defined for every Garside group. The interest on $\mathcal{C}_{\mathrm{AL}}(A)$ lies on the fact that it 
is $\delta$-hyperbolic when $A$ is irreducible. Calvez and Wiest conjectured that $\mathcal{C}_{\mathrm{AL}}\left(A_{n}\right)$ is quasi-isometric to the curve graph (the 1-skeleton of the curve complex) of $\mathcal{D}_{n}$ and they even made a step forward, proving that the braids that act loxodromically on $\mathcal{C}_{\mathrm{AL}}\left(A_{n}\right)$ are pseudo-Anosov. We recall that an element $\alpha$ acts loxodromically on $\mathcal{C}_{\mathrm{AL}}\left(A_{n}\right)$ if the orbit of every element of $\mathcal{C}_{\mathrm{AL}}(A)$ by $\alpha$ is quasi-isometric to $\mathbb{Z}$. The element $\alpha$ acts elliptically on $\mathcal{C}_{\mathrm{AL}}\left(A_{n}\right)$ if the orbits are bounded.

Proposition 1 [5, Proposition 2] We consider the action of the braid group $A_{n}$ on its additional length graph $\mathcal{C}_{\mathrm{AL}}\left(A_{n}\right)$ by left multiplication. Then periodic and reducible elements act elliptically.

On the other hand, we have the complex of irreducible parabolic subgroups $\mathcal{P}(A)$, defined by Cumplido, Gebhardt, González-Meneses and Wiest [9]. A standard parabolic subgroup, $A_{X}$, is a subgroup generated by some $X \subseteq \Sigma$. A subgroup $P$ is called parabolic if it is conjugate to a standard parabolic subgroup, that is, $P=\alpha^{-1} A_{Y} \alpha$ for some standard parabolic subgroup $A_{Y}$ and some $\alpha \in A$. We say that $P$ is irreducible, if it cannot be decomposed as a direct product of parabolic subgroups. Given an irreducible parabolic subgroup $P$, we denote by $z_{P}$ the unique positive element that generates the centre $Z(P)$ of $P$. The vertices of $\mathcal{P}(A)$ are the irreducible ones, that is, the parabolic subgroups that cannot be decomposed as a direct product of nontrivial parabolic subgroups. A set of vertices $\left\{P_{0}, \ldots, P_{n}\right\}$ spans an $n$-simplex if $z_{P_{i}} z_{P_{j}}=z_{P_{j}} z_{P_{i}}$ for all $i \neq j$. In [9, Theorem 2.2], it is proven that having $z_{P_{i}} z_{P_{j}}=z_{P_{j}} z_{P_{i}}$ is equivalent to have one of these three situations:

- $P_{i} \subset P_{j}$.

- $P_{j} \subset P_{i}$.

- $\quad P_{i} \cup P_{j}=\{1\}$ and $p_{i} p_{j}=p_{j} p_{i}$ for every $p_{i} \in P_{i}$ and $p_{j} \in P_{j}$.

In the braid case, the subset of irreducible proper parabolic subgroups is in bijection with the isotopy class of curves in $\mathcal{D}_{n}$. This makes $\mathcal{P}\left(A_{n}\right)$ isomorphic to the curve complex of $\mathcal{D}_{n}$. This complex seems then more natural for studying Artin-Tits groups than $\mathcal{C}_{\mathrm{AL}}(A)$. However, the hyperbolicity of the complex of irreducible parabolic subgroups is not proven yet. One approach to solve that problem could be to find links between the 1-skeleton of the complex of irreducible parabolic subgroups (which is in fact a flag complex) and the additional length graph, which is precisely what we will do in this article. We will generalize Proposition 1 of Calvez and Wiest to irreducible Artin-Tits groups of spherical type. Due to the bijection between the curve 
complex and the complex of irreducible parabolic subgroups mentioned before, we can realize that the reducible braids correspond to elements that preserve a family of parabolic subgroups that form a simplex in $\mathcal{P}(A)$. Notice that the action of $A$ on $\mathcal{P}(A)$ is induced by the conjugation action of $A$ on itself. We say that $x \in A$ is periodic if some power of it acts trivially on $\mathcal{P}(A)$.

Theorem 2 Let $A \neq A_{1}, A_{2}, I_{2 m}$ be an irreducible Artin-Tits group of spherical type. The periodic elements of $A$ and the elements preserving some simplex of $\mathcal{P}(A)$ (ie normalizing parabolic subgroup of $A$ ) act elliptically on $\mathcal{C}_{\mathrm{AL}}(A)$.

As explained by Calvez and Wiest [6, Proposition 4.9], the proof of this result (and more precisely Corollary 23) is the key to proving that there is a 9-Lipschitz function from $\mathcal{P}(A)$ to $\mathcal{C}_{\mathrm{AL}}(A)$ when $A$ has rank at least 3 . As a consequence, we can prove that in these cases $\mathcal{P}(A)$ has infinite diameter [6, Corollary 4.10].

For the second part of this article, we will use one of the key features of the graph $\mathcal{C}_{\mathrm{AL}}(A)$ : its hyperbolicity constant is independent of the Artin-Tits group of spherical type. Moreover, when proving Theorem 2 we will find bounds on the diameter of orbits of parabolic subgroups acting on $\mathcal{C}_{\mathrm{AL}}(A)$ that are again independent of the group $A$. Combining this uniformity of constants and standard techniques of groups acting on hyperbolic spaces, we will show that we can find a common "free-product complement" for all standard proper parabolic subgroups. Namely:

Theorem 3 There exists a constant $K$ such that for every irreducible Artin-Tits group $(A, \Sigma)$ of spherical type $A \neq A_{1}, A_{2}, I_{2 m}$, there is an element $g_{*} \in A^{+}$such that

(1) the element $g_{*}$ has length at most $K$ with respect to the Garside generators (see Section 2.1), and

(2) for every proper standard parabolic subgroup $A_{X}$ of $A$, one has that $\left\langle g_{*}, A_{X}\right\rangle \cong$ $\left\langle g_{*}\right\rangle * A_{X}$.

The Cayley graph of an Artin-Tits group of spherical type is usually better understood with respect to the Garside generators (explained in Section 2.1) than with respect to the Artin generating set $\Sigma$. Let $\mathcal{S}$ denote the set of Garside generators. Then, for every parabolic subgroup $A_{X}$, one has that the natural subgroup inclusion induces a graph isometric inclusion $\Gamma\left(A_{X}, A_{X} \cap \mathcal{S}^{ \pm 1}\right) \rightarrow \Gamma\left(A, \mathcal{S}^{ \pm 1}\right)$. Here, $\Gamma(G, X)$ denotes the Cayley graph of a group $G$ with respect to a generating set $X$. We will use this 
isometric inclusion together with the "free-product complement" to derive that the (relative) growth rate of proper parabolic subgroups is strictly smaller than the ambient group.

Before stating this last result, let us fix some notation. Let $M$ be a monoid and $X$ a finite generating set of $M$. We denote the (relative) exponential growth rate of $M$ by

$$
\omega(M, X)=\lim _{n \rightarrow \infty}\left(\#\left\{g \in M:|g|_{X} \leq n\right\}\right)^{1 / n},
$$

where $|g|_{X}$ denotes the length of the shortest word in $X$ representing $g$. Notice that this limit exists thanks to the submultiplicativity of the word length and Fekete's lemma.

Corollary 4 Let $A \neq A_{1}$ be an irreducible Artin-Tits group of spherical type. Let $\mathcal{S}_{A}$ be the Garside generating set of $A$. For every proper parabolic subgroup $A_{X}$ of $A$, one has that

$$
\omega\left(A_{X}, \mathcal{S}_{A}^{ \pm 1}\right)<\omega\left(A, \mathcal{S}_{A}^{ \pm 1}\right) \quad \text { and } \quad \omega\left(A_{X}^{+}, \mathcal{S}_{A}\right)<\omega\left(A^{+}, \mathcal{S}_{A}\right) .
$$

Moreover, the sequences $\left\{\omega\left(A_{n}, \mathcal{S}_{A_{n}}^{ \pm 1}\right)\right\}_{n=1}^{\infty},\left\{\omega\left(B_{n}, \mathcal{S}_{B_{n}}^{ \pm 1}\right)\right\}_{n=2}^{\infty},\left\{\omega\left(D_{n}, \mathcal{S}_{D_{n}}^{ \pm 1}\right)\right\}_{n=3}^{\infty}$ (and the corresponding sequences for the submonoid of positive elements) are increasing and unbounded.

This result contrasts with the case of standard Artin generators. In that case, it is known that both $\left\{\omega\left(A_{n}^{+}, \Sigma\right)\right\}_{n=1}^{\infty}$ and $\left\{\omega\left(A_{n}, \Sigma\right)\right\}_{n=1}^{\infty}$ are increasing and converge. More specifically, for the submonoid of positive elements, a beautiful recent result of Flores and González-Meneses [14, Theorem 6.8] shows that $\left\{\omega\left(A_{n}^{+}, \Sigma\right)\right\}_{n=1}^{\infty}$ converges to the KLV constant $q_{\infty}=3.23363 \ldots$

\section{Preliminaries}

\subsection{Garside theory}

Let us briefly recall some concepts from Garside theory (for a general reference, see [11]). A group $G$ is called a Garside group with Garside structure $(G, \mathcal{M}, \Delta)$ if it admits a submonoid $\mathcal{M}$ of positive elements such that $\mathcal{M} \cap \mathcal{M}^{-1}=\{1\}$ and a special element $\Delta \in \mathcal{M}$, called the Garside element, with the following properties:

- There is a partial order in $G$, $\preccurlyeq$, defined by $a \preccurlyeq b \Longleftrightarrow a^{-1} b \in \mathcal{M}$ such that for all $a, b \in G$ there exists a unique gcd, denoted by $a \wedge b$, and a unique $1 \mathrm{~cm}$, denoted by $a \vee b$, with respect to $\preccurlyeq$. This order is called prefix order and it is invariant under left-multiplication. 
- The set of simple elements $\mathcal{S}:=\{s \in G \mid 1 \preccurlyeq s \preccurlyeq \Delta\}$ generates $G$. These are also called Garside generators.

- $\Delta^{-1} \mathcal{M} \Delta=\mathcal{M}$.

- $\mathcal{M}$ is atomic: if we define the set of atoms as the set of elements $a \in \mathcal{M}$ such that there are no nontrivial elements $b, c \in \mathcal{M}$ such that $a=b c$, then for every $x \in \mathcal{M}$ there is an upper bound on the number of atoms in a decomposition of the form $x=a_{1} a_{2} \cdots a_{n}$, where each $a_{i}$ is an atom.

In a Garside group, the monoid $\mathcal{M}$ also induces a partial order which is invariant under right-multiplication, the suffix order $\succcurlyeq$. This order is defined by $a \geqslant b \Longleftrightarrow a b^{-1} \in \mathcal{M}$, and for all $a, b \in G$ there exists a unique gcd, $a \wedge^{\uparrow} b$, and a unique $1 \mathrm{~cm}, a \vee^{\natural} b$, with respect to $\succcurlyeq$.

We say that a Garside group has finite type if $\mathcal{S}$ is finite. It is well known that every Artin-Tits group of spherical type $A$ admits a Garside structure of finite type where the monoid $\mathcal{M}$ is precisely $A^{+}[3 ; 11]$. The monoid $A^{+}$injects on $A$ [21], which implies that the atoms of $A$ are precisely the generators in the presentation given in the introduction.

Remark 5 The conjugate by $\Delta$ of an element $x$ will be denoted by $\tau(x)=\Delta^{-1} x \Delta$. Notice that $\Delta^{-1} \mathcal{M} \Delta=\mathcal{M}$ implies that the set of prefixes of $\Delta$ equals its set of suffixes and then $\Delta$ is decomposed as $a \cdot b$, where $a$ is an atom, if and only if we can write $\Delta=b \cdot a^{\prime}$, where $a^{\prime}$ is an atom. This means that $\tau$ provides a permutation of the atoms of the Garside group.

Proposition 6 [3, Lemma 5.1, Theorem 7.1] Let $(\Sigma, A)$ be an Artin-Tits system of spherical type. Then the Garside element for $A$ is

$$
\Delta=\bigvee_{\sigma_{i} \in \Sigma}\left(\sigma_{i}\right)=\bigvee_{\sigma_{i} \in \Sigma}^{4}\left(\sigma_{i}\right)
$$

Moreover, the conjugation by $\Delta^{2}$ is trivial, that is, $\tau^{2}=\mathrm{Id}$.

Lemma 7 Let $x$ be an element of an Artin-Tits system $(A, \Sigma)$ of spherical type. Let

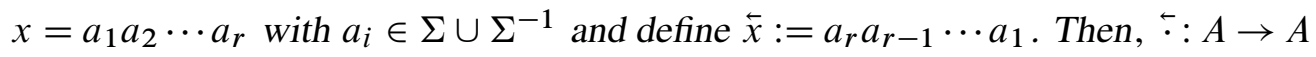
is an involution and a well-defined antihomomorphism (for all $x, y \in A \overleftarrow{x y}=\overleftarrow{y} \overleftarrow{x}$ ). In particular, $\Delta=\overleftarrow{\Delta}$.

Proof We have a well-defined antihomomorphism because the relations in the presentation of an Artin-Tits group of spherical type are symmetric, and so, for every 


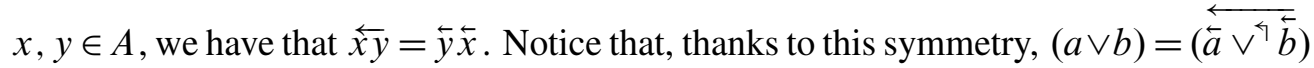
for every $a, b \in A^{+}$. Then, since our antihomomorphism preserves atoms,

$$
\bigvee_{\sigma_{i} \in \Sigma}\left(\sigma_{i}\right)=\left(\overleftarrow{V_{\sigma_{i} \in \Sigma}^{\sharp}\left(\overleftarrow{\sigma}_{i}\right)}\right)=\left(\overleftarrow{\bigvee_{\sigma_{i} \in \Sigma}^{\sharp}\left(\sigma_{i}\right)}\right)
$$

This fact together with Proposition 6 implies that $\Delta=\overleftarrow{\Delta}$.

Definition 8 We say that the product of two simple elements $a \cdot b$, is left-weighted (resp. right-weighted) if $a b \wedge \Delta=a$ (resp. $a b \wedge^{\uparrow} \Delta=b$ ).

Remark 9 In an Artin-Tits group of spherical type, the simple elements of the Garside structure are the square-free ones, that is, every positive word representing that element does not contain the square of an atom [3;12]. This implies that $a \cdot b$ is left-weighted (resp. right-weighted) if for every atom $t$ such that $t \preccurlyeq b$ (resp. $a \succcurlyeq t$ ), we have that $a \succcurlyeq t$ (resp. $t \preccurlyeq b)$.

Definition 10 We say that $x=\Delta^{k} s_{1} \cdots s_{r}$ is in left normal form if $k \in \mathbb{Z}, s_{i} \notin\{1, \Delta\}$ is a simple element for $i=1, \ldots, r$, and $s_{i} \cdot s_{i+1}$ is left-weighted for $0<i<r$. Analogously, $x=s_{1} \cdots s_{r} \Delta^{k}$ is in right normal form if $k \in \mathbb{Z}, s_{i} \notin\{1, \Delta\}$ is a simple element for $i=1, \ldots, r$, and $s_{i} s_{i+1}$ is right-weighted for $0<i<r$. When the right and the left normal form coincide, we will just refer to the normal form.

It is well known that the normal forms of an element are unique [11, Corollary 7.5] and that the numbers $r$ and $k$ do not depend on the normal form (left or right). We define the infimum, the canonical length and the supremum of $x$, respectively, as $\inf (x)=k$, $\ell(x)=r$ and $\sup (x)=k+r$. Equivalents definitions of supremum and infimum are

$$
\inf (x)=\max \left\{p \mid \Delta^{p} \preccurlyeq x\right\} \quad \text { and } \quad \sup (x)=\min \left\{p \mid x \preccurlyeq \Delta^{p}\right\} .
$$

\subsection{The additional length graph}

The construction of the additional length graph is made for any Garside group and its key ingredient is the use of absorbable elements, which are defined below.

Definition 11 [5, Definition 1] Let $G$ be a Garside group. We say that $y \in G$ is an absorbable element if the two following conditions are satisfied:

(1) $\inf (y)=0$ or $\sup (y)=0$.

(2) There is an $x \in G$ such that $\inf (x y)=\inf (x)$ and $\sup (x y)=\sup (x)$.

In this case we say that $x$ absorbs $y$. 
We say that $x$ absorbs $y$ because the length of the normal form of $x y$ is the same length of the normal form of $x$. So, loosely speaking, the normal form of $x$ "absorbs" the factors of the normal form of $y$.

Example Consider the braid group $A_{n}$ with $n>2$. As $\sigma_{1}$ commutes with $\sigma_{3}$, if we let $x=\sigma_{1} \cdots \sigma_{1}$ and $y=\sigma_{3} \cdots \sigma_{3}$, we have that $x y=\left(\sigma_{1} \sigma_{3}\right) \cdots\left(\sigma_{1} \sigma_{3}\right)$. Hence, $x$ absorbs $y$.

On the other hand, if $x=\Delta \sigma_{i}^{-1}$ and $y=\sigma_{i}$, then $x$ does not absorb $y$ because $\inf (x)=0$ and $\inf (x y)=1$.

Definition 12 [5, Definition 2] Let $\left(G, G_{+}, \Delta\right)$ be a Garside structure. We define the additional length graph of $G, \mathcal{C}_{\mathrm{AL}}(G)$ as follows:

- The vertices are in one-to-one correspondence with $G /\langle\Delta\rangle$, that is, the equivalence classes $g \Delta^{\mathbb{Z}}=\left\{g \Delta^{p} \mid p \in \mathbb{Z}\right\}$. Every class $v$ has a unique representative with infimum 0 , denoted by $\bar{v}$.

- Two vertices $v=\bar{v} \Delta^{\mathbb{Z}}$ and $w=\bar{w} \Delta^{\mathbb{Z}}$ are connected by an edge if and only if we have one of the two following situations:

(1) There is a simple element $m \neq 1, \Delta$ such that $\bar{v} m \in w$.

(2) There is an absorbable element $y \in G$ such that $\bar{v} y \in w$.

We give a metric structure to this complex by saying that the length of every edge in the graph is 1 . We denote the distance between two vertices $v$ and $w$ by $\mathrm{d}_{\mathcal{C}_{\mathrm{AL}}}(v, w)$.

\section{Normalizers of parabolic subgroups act elliptically}

The aim of this section is to prove Theorem 2, that is, we prove that the normalizers of parabolic subgroups of an irreducible spherical Artin-Tits group $A \neq A_{1}, A_{2}, I_{2 m}$ act elliptically on the additional length graph.

In [19] it is proven that any standard parabolic subgroup $A_{X}$ of an Artin-Tits group of spherical type is an Artin-Tits group of spherical type itself. This means that $A_{X}$ has also a Garside structure, whose Garside element is denoted by $\Delta_{X}$ and equals the least common multiple of the elements in $X$. We denote by $\tau_{X}$ the conjugation by $\Delta_{X}$. 


\subsection{Ribbons}

We will use some objects defined in [8;9] that we call ribbons. We shall remark that these ribbons are slightly different from the classical concept of ribbon introduced in [16].

Definition 13 Let $(A, \Sigma)$ be an Artin-Tits system of spherical type and let $X \subsetneq \Sigma$ and $t \in \Sigma$. We define

$$
r_{X, t}=\Delta_{X \cup\{t\}} \Delta_{X}^{-1}, \quad r_{t, X}=\tau_{X \cup\{t\}}\left(r_{X, t}\right)=\Delta_{X}^{-1} \Delta_{X \cup\{t\}} .
$$

We will say that $r_{X, t}$ is a right-ribbon and $r_{t, X}$ is a left-ribbon.

Remark 14 Notice that $\Delta_{X \cup\{t\}}=r_{X, t} \Delta_{X}$ is simple and so it is square-free (Remark 9). As $\Delta_{X}$ can start with any letter of $X$, if $t \notin X$, the only suffix letter of $r_{X, t}$ is $t$. Analogously, $t$ is the only prefix letter of $r_{t, X}$.

Lemma 15 Let $(A, \Sigma)$ be an Artin-Tits system of spherical type and let $X \subset \Sigma$. Then $r_{t, X}=\overleftarrow{r_{X, t}}$. In particular, if $t \notin X$, then both $r_{X, t} \cdot r_{t, X}$ and $r_{t, X} \cdot r_{X, t}$ are leftand right-weighted.

Proof By Lemma 7, we have that

$$
\overleftarrow{r_{X, t}}=\overleftarrow{\Delta_{X}^{-1}} \cdot \overleftarrow{\left.\Delta_{X \cup\{t}\right\}}=\Delta_{X}^{-1} \Delta_{X \cup\{t\}}=r_{t, X}
$$

Also notice that, as we have seen in that lemma, the atoms that are suffixes of $x$ coincide with the atoms that are prefixes of $\bar{x}$ and vice versa. Then, by Remark $9, r_{X, t} \cdot r_{t, X}$ and $r_{t, X} \cdot r_{X, t}$ are both left- and right-weighted.

Remark 16 By definition, conjugations by $r_{X, t}$ and $r_{t, X}$ are equivalent to applying $\tau_{X} \circ \tau_{X \cup\{t\}}$ and $\tau_{X \cup\{t\}} \circ \tau_{X}$, respectively (recall that $\tau^{2}=\mathrm{Id}$ ). So, by Remark 5, conjugation by $r_{X, t}$ and $r_{t, X}$ induces a permutation of the atoms of $X \cup\{t\}$. This implies that there exists a unique $Y \subset X \cup\{t\}$ such that $r_{X, t} X=Y r_{X, t}$ and $X r_{t, X}=r_{t, X} Y$. We say that $r_{X, t}$ is an elementary $X-$ ribbon $-Y$.

Also, since $t$ is the only atomic prefix of $r_{t, X}$ (Remark 14), $r_{X, t} s$ is simple for every $s \in X$. So $Y$ is formed by all atoms $u \in X \cup\{t\}$ such that $u \nless r_{X, t}$ and $r_{t, X} \nsucceq u$ (Remark 9). Moreover, $r_{X, t}$ has a unique atomic prefix and $r_{t, X}$ has a unique atomic suffix. 
Definition 17 Let $(A, \Sigma)$ be an Artin-Tits system of spherical type and $X, Y \subsetneq \Sigma$. We say that $\alpha \in A$ is an $X$-ribbon $-Y$ if $\alpha$ can be decomposed as a product of left-ribbons $r_{1} \cdots r_{m}$ and there exists a sequence of subsets of $\Sigma$ of the form $X_{1}=X, X_{2}, \ldots, X_{m}$, $X_{m+1}=Y$ such that $r_{i}$ is an elementary $X_{i}$-ribbon- $X_{i+1}$.

\subsection{Proof of Theorem 2}

We will prove that we can write any normalizer of a proper standard parabolic subgroup as the product of at most nine absorbable elements.

Lemma 18 Let $(A, \Sigma)$ with $A \neq A_{1}, A_{2}, I_{2 m}$ be an Artin-Tits system of spherical type with Garside element $\Delta$ and let $X \subsetneq \Sigma$. Then, for every $k \in \mathbb{Z}, \Delta^{k}$ is a product of at most three absorbable elements and $\Delta_{X}^{k}$ is a product of at most two absorbable elements.

Proof Suppose that $k>0$. Take $A=\sigma_{i}^{k}$ and $B=\sigma_{j}^{k}$, where $\sigma_{i} \in \Sigma$ and $\sigma_{j} \in \Sigma$ commute, and $C=B^{-1} \cdot A^{-1} \cdot \Delta^{k}$. We claim that $A, B$ and $C$ are absorbable. If this is true, $A B C$ is the desired decomposition for $\Delta^{k}$. Also, by [5, Lemma 1], $C^{-1} \cdot B^{-1} \cdot A^{-1}$ is the desired decomposition of $\Delta^{k}$ when $k<0$.

Firstly, we have that $\inf (A)=\inf (B)=0$. We want to see that also $\inf (C)=0$. As $A \cdot B=\left(\sigma_{i} \sigma_{j}\right)^{k}$, we can write

$$
C=\Delta \tau\left(\left(\sigma_{i} \sigma_{j}\right)^{-1}\right) \cdot \Delta \tau^{2}\left(\left(\sigma_{i} \sigma_{j}\right)^{-1}\right) \cdots \Delta \tau^{k}\left(\left(\sigma_{i} \sigma_{j}\right)^{-1}\right) .
$$

Notice that $\Delta \tau^{p+1}\left(\left(\sigma_{i} \sigma_{j}\right)^{-1}\right)=\tau^{p}\left(\left(\sigma_{i} \sigma_{j}\right)^{-1}\right) \Delta=\tau^{p}\left(\left(\sigma_{j} \sigma_{i}\right)^{-1}\right) \Delta$. By Lemma 7 , we have that for every $q>0$ and every atom $\sigma_{m}$,

$$
\Delta \tau^{q}\left(\left(\sigma_{i} \sigma_{j}\right)^{-1}\right) \succcurlyeq \sigma_{m} \Longleftrightarrow \sigma_{m} \preccurlyeq \tau^{q}\left(\left(\sigma_{j} \sigma_{i}\right)^{-1}\right) \Delta,
$$

meaning that $\Delta \tau^{p-1}\left(\left(\sigma_{i} \sigma_{j}\right)^{-1}\right) \cdot \Delta \tau^{p}\left(\left(\sigma_{i} \sigma_{j}\right)^{-1}\right)$ is left- and right-weighted for every $p>0$. Hence, (1) is the normal form of $C$ and $\inf (C)=0$.

We can easily see that $A$ and $B$ absorb each other. Now let us see that $B$ absorbs $C$. We have that

$$
B \cdot C=\left(\sigma_{i}\right)^{-k} \cdot \Delta^{k}=\Delta \tau\left(\sigma_{i}^{-1}\right) \cdot \Delta \tau^{2}\left(\sigma_{i}^{-1}\right) \cdots \Delta \tau^{k}\left(\sigma_{i}^{-1}\right) .
$$

As before, this expression is the normal form of $B \cdot C$. Then $\inf (B)=\inf (B \cdot C)=0$ and $\sup (B)=\sup (B \cdot C)=k$, as desired. This concludes that $\Delta^{k}$ is a product of at most three absorbable elements. 
In most of the cases, $\Delta_{X}^{k}$ is absorbable itself. It suffices to have an atom $\sigma_{j}$ that commutes with $X$, so that $\sigma_{j}^{k}$ absorbs $\Delta_{X}^{k}$. If this is not the case, we can take $\sigma_{i} \in X$ and $\sigma_{j} \notin X$ such that $\sigma_{i} \sigma_{j}=\sigma_{j} \sigma_{i}$ and let $A=\sigma_{i}^{k}$ and $B=A^{-1} \Delta_{X}^{k}$. Then $A$ is absorbed by $\sigma_{j}^{k}$ and $A$ absorbs $B$.

Proposition 19 Let $A \neq A_{1}, A_{2}, I_{2 m}$ be any Artin-Tits group of spherical type. Every element in a proper standard parabolic subgroup of $A$ is a product of at most three absorbable elements. In particular, the orbit on $\mathcal{C}_{\mathrm{AL}}(A)$ of every proper standard parabolic subgroup of $A$ has diameter at most 3.

Proof Let $A_{X}$ be a proper standard parabolic subgroup of $A$ and take $x \in A_{X}$. As $A_{X}$ is an Artin-Tits group of spherical type, we can take the left normal form of $x$ in $A_{X}$, which is of the form $\Delta_{X}^{k} s_{1} \cdots s_{l}$. By Lemma $18, \Delta_{X}^{k}$ is a product of at most two absorbable elements. So, we assume that $x=s_{1} \cdots s_{l}$. We want to see that this element is absorbable.

If there is $\sigma_{i} \in \Sigma \backslash X$ that commutes with $X$, then $\sigma_{i}^{l}$ absorbs $s_{1} \cdots s_{l}$. Otherwise, take an atom $t \in \Sigma \backslash X$ not commuting with $X$. We claim that

$$
y=\tau_{X \cup\{t\}}^{l-1}\left(r_{X, t}\right) \cdot \tau_{X \cup\{t\}}^{l-2}\left(r_{X, t}\right) \cdots \tau_{X \cup\{t\}}\left(r_{X, t}\right) \cdot r_{X, t}
$$

absorbs $s_{1} \cdots s_{l}$. Firstly, recall that $\tau_{X \cup\{t\}}\left(r_{X, t}\right)=r_{t, X}$ and that, by Lemma 15, the expression (2) is the normal formal of $y, \operatorname{so} \inf (y)=0$ and $\sup (y)=l$. As conjugations by $r_{X, t}^{-1}$ and $r_{t, X}^{-1}$ are equivalent to applying $\tau_{X \cup\{t\}} \circ \tau_{X}$ and $\tau_{X} \circ \tau_{X \cup\{t\}}$, respectively, conjugation by $r_{X, t}^{-1} r_{t, X}^{-1}$ fixes every element in $A_{X}$. This allows us to write

$$
y x=s_{l}^{\prime} \cdot s_{l-1}^{\prime} \cdots s_{1}^{\prime},
$$

where $s_{i}^{\prime}=r_{X, t} \cdot s_{l-i+1}$ if $i$ is odd and $s_{i}^{\prime}=s_{l-i+1} \cdot r_{t, X}$ if $i$ is even. We want to prove that (3) expresses the normal form of $y x$ and $s_{i}^{\prime} \neq \Delta$ for $1 \leq i \leq l$. To do that, it suffices to show the following:

(1) $s r_{t, X}$ and $r_{X, t} s$ are simple and different from $\Delta$ for every $s \preccurlyeq \Delta_{X}, s \neq \Delta_{X}$.

(2) If $a \cdot b$ is left-weighted for $a, b \preccurlyeq \Delta_{X}$, then $a r_{t, X} \cdot r_{X, t} b$ and $r_{X, t} a \cdot b r_{t, X}$ are left-weighted.

To prove the first statement, notice that there exists a simple element $s^{\prime} \in \Delta_{X}$ such that $s s^{\prime}=\Delta_{X}$. On the other hand, by definition, $\Delta_{X \cup\{t\}}=\Delta_{X} r_{t, X}=s s^{\prime} r_{t, X}$. By Remark 16, there is a positive element $s^{\prime \prime} \in \Delta_{X \cup\{t\}}$ such that $s^{\prime} r_{t, X}=r_{t, X} s^{\prime \prime}$, hence 
$\Delta_{X \cup\{t\}}=s r_{t, X} s^{\prime \prime}$. Hence $s r_{t, X}$ is simple, which is different from $\Delta$ because $s \neq \Delta_{X}$. For $r_{X, t} s$, the reasoning is analogous, using that $\Delta_{X} \geqslant s$.

Let us now prove the second statement. Let $u \in X \cup\{t\}$ be the only atomic suffix of $r_{t, X}$ (Remark 16). By Lemma 15, $u \preccurlyeq r_{X, t}$, so take an atom $u^{\prime} \neq u$ such that $u^{\prime} \preccurlyeq r_{X, t} b$. By Remark 16, we have $u^{\prime} r_{X, t}=r_{X, t} \tau_{X}\left(\tau_{X \cup\{t\}}\left(u^{\prime}\right)\right)=u^{\prime} \vee r_{X, t} \preccurlyeq r_{X, t} b$, which means that $\tau_{X}\left(\tau_{X \cup\{t\}}\left(u^{\prime}\right)\right) \preccurlyeq b$. But, as $a \cdot b$ is left-weighted, this implies that $a \geqslant \tau_{X}\left(\tau_{X \cup\{t\}}\left(u^{\prime}\right)\right)$, which implies $a r_{t, X} \geqslant u^{\prime}$. This proves that $a r_{t, X} \cdot r_{X, t} b$ is left-weighted.

For the other product, notice that

$$
r_{X, t} a=\tau_{X \cup\{t\}}\left(\tau_{X}(a)\right) r_{X, t} \quad \text { and } \quad b r_{t, X}=r_{t, X} \tau_{X \cup\{t\}}\left(\tau_{X}(b)\right) .
$$

As $r_{X, t} \cdot r_{t, X}$ is left-weighted (Lemma 15) and the permutation induced by $\tau_{X \cup\{t\}} \circ \tau_{X}$ preserves normal forms for elements in $A_{X}, \tau_{X \cup\{t\}}\left(\tau_{X}(a)\right) \cdot \tau_{X \cup\{t\}}\left(\tau_{X}(b)\right)$ is also left-weighted, and we can apply the same arguments as above.

Therefore, we have proved that $\inf (y x)=\inf (y)=0$ and $\sup (y x)=\sup (y)=l$, as we wanted to show.

Remark 20 Let us see why the later results do not work for $A_{1}, A_{2}$ and $I_{2 m}$. Notice that, by definition, any absorbable element lies in $A^{+}$or in $A^{-}$(the negative monoid of $A$ ). We also recall that the only simple elements that are not absorbable are the ones of the form $\sigma_{i}^{-1} \Delta$ [5, Example 1]. For $A_{1}$ the set of absorbable elements is trivial and for $A_{2}$ the only absorbable elements are $\sigma_{1}, \sigma_{1}^{-1}, \sigma_{2}$ and $\sigma_{2}^{-1}$, so $x$ cannot be obtained as product of fewer than $3 \cdot|x|$ absorbable elements. Here $|\cdot|$ denotes the word length with respect to the set of standard Artin generators, $\Sigma$.

On the other hand, the only absorbable elements in $I_{2 m}$ are the absorbable simple elements and their inverses, that is, the elements of the form

$$
\underbrace{\sigma_{1} \sigma_{2} \sigma_{1} \cdots}_{p \text { elements }} \text { or } \underbrace{\sigma_{2} \sigma_{1} \sigma_{2} \cdots}_{p \text { elements }}
$$

with $p<m-1$ and their inverses. To see that no other element is absorbable, take an element in $I_{2 m}$ of infimum 0 whose normal form is $s_{1} \cdot s_{2}$. By [5, Lemma 3] if $s_{1} \cdot s_{2}$ is absorbable, then it is absorbed by an element of infimum 0 with normal form $s_{1}^{\prime} \cdot s_{2}^{\prime}$. Let $s_{1}^{\prime} \succcurlyeq \sigma_{i} \preccurlyeq s_{2}^{\prime}$ and $s_{1} \succcurlyeq \sigma_{j} \preccurlyeq s_{2}$. If we suppose that $\sigma_{1} \preccurlyeq s_{1}$ (the case with $\sigma_{2}$ is analogous), then, by absorbability, $s_{2}^{\prime} \geqslant \sigma_{2}$. If $s_{2}^{\prime} s_{1}$ were not simple, we would have that $\inf \left(s_{1}^{\prime} \cdot s_{2}^{\prime} \cdot s_{1} \cdot s_{2}\right)>0$, contradicting absorbability. Hence, let us assume that $s_{2}^{\prime} s_{1}$ 
is a simple element of the form $\sigma_{i} \cdots \sigma_{2} \sigma_{1} \cdots \sigma_{j}$. This implies that the normal form of $s_{1}^{\prime} \cdot s_{2}^{\prime} \cdot s_{1} \cdot s_{2}$ is $s_{1}^{\prime} \cdot s_{2}^{\prime} s_{1} \cdot s_{2}$ of length 3 , which also contradicts absorbability. Therefore, the length of the minimal expression of $x$ as a product of absorbable elements in $I_{2 m}$ depends on $|x|$.

The support of a positive element $u$, denoted by $\operatorname{supp}(u)$, is the set of generators that appear in every positive word representing $u$. Notice that $\operatorname{supp}\left(\Delta_{X}\right)=X$ for any $X \subseteq \Sigma$. The next lemma is a generalization of [20, Lemma 5.6; 16, Lemma 2.2], which use the classical concept of ribbon.

Lemma 21 Let $(A, \Sigma)$ be an Artin-Tits system of spherical type, $X, Y \subsetneq \Sigma$ and $u, v \in A^{+}$with $\operatorname{supp}(u)=X$ and $\operatorname{supp}(v)=Y$. Then any element $z \in A^{+}$such that $z^{-1} u z=v$ can be written as $z=\alpha \beta$, where $\alpha \in A_{X}$ and $\beta$ is an $X$-ribbon- $Y$.

Proof We will use [9, Proposition 6.3], which says that if $c \preccurlyeq z$ is a minimal element conjugating $u$ to a positive element (meaning that there is no $c^{\prime} \preccurlyeq c$ with $c^{\prime} \neq 1, c$ such that $c^{\prime-1} u c^{\prime} \in A^{+}$), then either $c \in A_{X}$, or $c=r_{t, X}$ for some $t \in \Sigma$ such that $t \notin X$. Using this, we can write $z$ as a product $c_{1} \cdots c_{r}$, where $c_{1}$ is a minimal conjugator from $v_{0}:=u$ to a positive element and $c_{i}$ is a minimal conjugator from $v_{i}:=\left(c_{1} \cdots c_{i-1}\right)^{-1} u\left(c_{1} \cdots c_{i-1}\right)$ to a positive element for $1<i \leq r$. If we let $Y_{i}=\operatorname{supp}\left(v_{i}\right)$, then either $c_{i} \in A_{Y_{i}}$ (type 1), or $c_{i}=r_{t, Y_{i}}$ for some $t \in \Sigma$ such that $t \notin Y_{i}$ (type 2).

Suppose that we have some $c_{i}$ of type 2 and $c_{i+1}$ of type 1 . In this case, $c_{i} c_{i+1}=$ $c_{i+1}^{\prime} c_{i}$, where $c_{i+1}^{\prime} \in A_{Y_{i}}$, having an element of type 1 before an element of type 2 . This allows us to arrange the product $c_{1} \cdots c_{r}$ to have $c_{1} \cdots c_{r}=\alpha \beta$, where $\alpha \in A_{X}$ and $\beta$ is an $X$-ribbon- $Y$, as we wanted.

Proposition 22 Let $(A, \Sigma)$ with $A \neq I_{2 m}, A_{1}, A_{2}$ be an Artin-Tits system of spherical type, $X \subsetneq \Sigma$ and $u \in A^{+}$with $\operatorname{supp}(u)=X$. Then any element $x \in A$ such that $x^{-1} u x \in A^{+}$is a product of at most nine absorbable elements.

Proof Let $\Delta^{k} x_{1} \cdots x_{r}$ be the left normal form of $x$. Notice that $x_{1} \cdots x_{r}$ is a positive element that conjugates $\tau^{k}(u) \in A^{+}$to a positive element. If we write $X^{\prime}=\operatorname{supp}\left(\tau^{k}(u)\right)$, by Lemma 21 we have the decomposition $x_{1} \cdots x_{r}=\alpha \cdot \beta$, with $\alpha \in A_{X^{\prime}}$ and $\beta$ an $X^{\prime}$-ribbon- $Y$, where $Y:=\operatorname{supp}\left(x^{-1} u x\right)$.

Suppose that $\beta$ is an $X^{\prime}$-ribbon- $Y$ of the form $r_{1} \cdots r_{m}$, satisfying $r_{i} \neq 1$ and $X_{i} r_{i}=r_{i} X_{i+1}$, where $X_{i} \subsetneq \Sigma$, for every $1 \leq i \leq m$. Thanks to [20, Theorem 5.1] 
and the proof of [15, Lemma 2.2] we know that if $Z_{1} s=s Z_{2}$ for some $Z_{1}, Z_{2} \subsetneq \Sigma$ and $s \in A$, then $\Delta_{Z_{1}} s=s \Delta_{Z_{2}}$. Hence, using the definition of left-ribbon we can write $\beta$ in the form $\Delta_{X}^{-m} \Delta_{X_{1} \cup\left\{t_{1}\right\}} \cdots \Delta_{X_{m} \cup\left\{t_{m}\right\}}$, where $t_{i} \in \Sigma \backslash X_{i}$ for every $1 \leq i \leq m$. Let $q \geq 0$ be the maximum number such that $\Delta^{q} \preccurlyeq \Delta_{X_{1} \cup\left\{t_{1}\right\}} \cdots \Delta_{X_{m} \cup\left\{t_{m}\right\}}$ and write $\beta^{\prime}:=\Delta_{X_{1} \cup\left\{t_{1}\right\}} \cdots \Delta_{X_{m} \cup\left\{t_{m}\right\}}=\Delta^{q} \gamma$ for some positive $\gamma$. By conjugating, we can "move" $\Delta^{q}$ to the left in order to have

$$
x=\Delta^{k+q} \cdot \tau^{q}(\alpha) \cdot \Delta_{Y^{\prime}}^{-m} \gamma,
$$

where $\Delta_{Y^{\prime}}=\tau^{q}\left(\Delta_{X^{\prime}}\right)$, so $Y^{\prime} \subsetneq \Sigma$. As $\tau^{q}(\alpha) \in A_{Y^{\prime}}$, by Lemma 18 and Proposition 19 the element $\Delta^{k+q} \cdot \tau^{q}(\alpha)$ is a product of at most six absorbable elements. Notice that $q \leq m$.

We claim that $\Delta_{Y^{\prime}}^{-m} \gamma$ is product of at most three absorbable elements. Observe that $\Delta^{q}$ and $\Delta_{X^{\prime}}^{m}$ are prefixes of $\beta^{\prime}$, hence $\Delta^{q} \vee \Delta_{X^{\prime}}^{m}=\Delta_{X^{\prime}}^{m-q} \Delta^{q}=\Delta^{q} \Delta_{Y^{\prime}}^{m-q}$ is a prefix of $\beta^{\prime}$. This means that $\Delta_{Y^{\prime}}^{m-q} \preccurlyeq \gamma$ and then $\inf \left(\Delta_{Y^{\prime}}^{q-m} \gamma\right)=0$. Then we decompose $\Delta_{Y^{\prime}}^{-m} \gamma=\Delta_{Y^{\prime}}^{-q} \cdot \Delta_{Y^{\prime}}^{q-m} \gamma$. We know that $\Delta_{Y^{\prime}}^{-q}$ is a product of at most two absorbable elements. Finally, we claim that $\Delta_{Y^{\prime}}^{m-q}$ absorbs $\Delta_{Y^{\prime}}^{q-m} \gamma$. Since, by construction, $\inf (\gamma)=0$, we just need to prove that $\sup (\gamma)=m-q$. Notice that $\Delta_{Y^{\prime}}^{m-q} \preccurlyeq \gamma$ and $\Delta_{Y^{\prime}}^{m-q} \nless \Delta^{p}$ for $1 \leq p \leq m-q-1$, hence $\sup (\gamma)$ is at least $m-q$. On the other hand, $\beta^{\prime} \preccurlyeq \Delta^{m}$, so $\gamma \preccurlyeq \Delta^{m-q}$ and $\inf (\gamma) \leq m-q$. Thus $\inf (\gamma)=m-q$, as we wanted to prove.

Corollary 23 Let $(A, \Sigma)$ with $A \neq I_{2 m}, A_{1}, A_{2}$ be an Artin-Tits system of spherical type and $X \subsetneq \Sigma$. The elements of $A$ normalizing $A_{X}$ are the product of at most nine absorbable elements.

Proof In [8, Lemma 7], it is proven that $\alpha \in A$ normalizes $A_{X}$ if $\alpha$ commutes with an element, called the central Garside element of $A_{X}$, which turns to be a positive power of $\Delta_{X}$. Therefore, Proposition 22 applies and $\alpha$ is the product of at most nine absorbable elements.

Proof of Theorem 2 We proceed as in the proof of [5, Proposition 2]. Firstly, notice that by definition some power of a periodic element $x$ acts trivially on $\mathcal{C}_{\mathrm{AL}}(A)$. This means that $x$ acts as finite-order isometries and then it acts elliptically on $\mathcal{C}_{\mathrm{AL}}(A)$.

If $y$ is an element normalizing some standard parabolic subgroup $A_{X}$, by Corollary 23 the orbit of the trivial element by the action of $y$ on $\mathcal{C}_{\mathrm{AL}}(A)$ remains at distance at 
most 9 from the trivial element ( $y^{p}$ normalizes $A_{X}$ for every $p>1$ ). Hence, $y$ acts elliptically on $\mathcal{C}_{\mathrm{AL}}(A)$. Finally, each element that normalizes the parabolic subgroup $P=\alpha^{-1} A_{X} \alpha$ for some $\alpha \in A$ is the conjugate of an element normalizing $A_{X}$, and therefore it also acts elliptically on $\mathcal{C}_{\mathrm{AL}}(A)$.

\section{Free-product complement}

In this section we prove Theorem 3, that is, the existence of a "free-product complement" for standard parabolic subgroups. The proof involves theory about groups acting on hyperbolic spaces.

\subsection{WPD elements and elementary subgroups}

Let $G$ be a group acting on a hyperbolic metric space $\mathcal{H}$ by isometries.

We say that $g \in G$ satisfies the WPD (weak proper discontinuity) condition if for every $\epsilon>0$ and every $v \in \mathcal{H}$, there exists $R=R(\epsilon)$ such that

$$
\#\left\{h \in G: \mathrm{d}_{\mathcal{H}}(v, h v) \leq \epsilon \text { and } \mathrm{d}_{\mathcal{H}}\left(g^{R} v, h g^{R} v\right) \leq \epsilon\right\}<\infty .
$$

According to [10, Lemma 6.5 and Corollary 6.6], for every WPD element $g \in G$ acting loxodromically, there exists a unique maximal virtually cyclic subgroup, denoted by $E_{G}(g)$, which consists of the elements that stabilize a quasigeodesic axis for $\langle g\rangle$. Moreover, it can be shown that

$$
E_{G}(g)=\left\{h \in G \mid h g^{i} h^{-1}=g^{j} \text { for some } i, j \in \mathbb{Z}\right\} .
$$

Notice that the torsion-free elements in $E_{G}(g)$ are also loxodromic. Otherwise, the WPD condition would not be satisfied.

The starting point for proving Theorem 3 is the hyperbolicity of $\mathcal{C}_{\mathrm{AL}}(A)$ proved by Calvez and Wiest. We summarize the facts in [5, Theorem 1; 4, Theorems 1 and 2, Propositions 5 and 6 and Remark 1] about $\mathcal{C}_{\mathrm{AL}}(A)$ in the next theorem:

Theorem 24 The additional length graph $\mathcal{C}_{\mathrm{AL}}(A)$ associated to the classical Garside structure of an irreducible Artin-Tits group of spherical type $A$ is 60-hyperbolic. Let $v=\langle\Delta\rangle$ be a vertex in $\mathcal{C}_{\mathrm{AL}}(A)$. There is an element $g \in A / Z(A)$ satisfying:

(i) $g$ has a preimage $\widetilde{g}$ in $A^{+}$of Garside length bounded by 12 , that is, $|\widetilde{g}|_{\mathcal{S}} \leq 12$.

(ii) $g$ acts loxodromically and $\mathrm{d}_{\mathcal{C}_{\mathrm{AL}}}\left(v, g^{n} v\right) \geq \frac{1}{2} n$. 
(iii) $g$ is WPD and, in particular, for every $\kappa>0$ there is $N=N(\kappa)=4 \kappa+319$ such that the cardinality of the set

$$
\left\{h \in A / Z(A): \mathrm{d}_{\mathcal{C}_{\mathrm{AL}}}(v, h v) \leq \kappa, \mathrm{d}_{\mathcal{C}_{\mathrm{AL}}}\left(g^{N} v, h g^{N} v\right) \leq \kappa\right\}
$$

is bounded above by $F(\kappa)=8 \kappa+638$.

It is extremely important for our applications to notice that the constants involved in Theorem 24 are independent of the Artin-Tits group $A$.

\subsection{A technical lemma}

The proof of Theorem 3 is a standard application of techniques of groups acting on hyperbolic spaces and it can be deduced easily from the results in [10]. However, it is not easy to trace back in the literature the exact dependency of the constants needed to have a unique constant $K$ in the statement of Theorem 3. For that reason we will repeat some well-known arguments. The main point is showing that "cancellations" between products of elements of $\left\langle g^{n}, P \cdot Z(A) / Z(A)\right\rangle \subseteq A$ can be uniformly controlled when $n$ is large enough.

Lemma 25 Let $A_{X}$ be a proper standard parabolic subgroup of an irreducible ArtinTits group $A \neq A_{1}, A_{2}, I_{2 m}$ of spherical type. Let $v=\langle\Delta\rangle$ be a vertex in $\mathcal{C}_{\mathrm{AL}}(A)$ and let $g$ be the element in Theorem 24. Denote by $\delta=60$ the hyperbolicity constant of $\mathcal{C}_{\mathrm{AL}}(A)$ and $\mathrm{d}:=\mathrm{d}_{\mathcal{C}_{\mathrm{AL}}}$. Let $a \geq 0$ and $n$ be big enough (only depending on $a, g$ and $\left.\mathcal{C}_{\mathrm{AL}}(A)\right)$. Then, for all $e, f \in\left\{g^{-1}, g\right\}$ and for any nontrivial element $t \in\left(A_{X} \cdot Z(A)\right) / Z(A)$, the following hold:

$$
\mathrm{d}\left(e^{n} v, t f^{n} v\right) \geq \max \left\{\mathrm{d}\left(v, e^{n} v\right), \mathrm{d}\left(v, t f^{n} v\right)\right\}+2 \delta+a .
$$

Proof We follow the argument of [2, Proposition 6]. In fact, what we are going to show is that if (4) does not hold for $n$ large enough, then $t \in E_{A / Z(A)}(g)$. Since $t$ stabilizes $A_{X}$ and $A_{X}$ is proper parabolic, Theorem 2 implies that $t$ acts elliptically on $\mathcal{C}_{\mathrm{AL}}(A)$. On the other hand, $t$ is an infinite-order element because $\langle\Delta\rangle \cap A_{X}=\{1\}$ and $t$ is a nontrivial element of $\left(A_{X} \cdot Z(A)\right) / Z(A)$. As $t$ lies in $E_{A / Z(A)}(g)$, we have that $t$ has to act loxodromically on $\mathcal{C}_{\mathrm{AL}}(A)$, which is a contradiction.

Notice that $\mathrm{d}\left(v, e^{n} v\right)=\mathrm{d}\left(v, f^{n} v\right)$ for any choice of $e, f \in\left\{g^{-1}, g\right\}$. Also, for any element $t \in\left(A_{X} \cdot Z(A)\right) / Z(A)$, we have that $\mathrm{d}(v, t v) \leq 9$ by Corollary 23. By the triangle inequality, we have that $\mathrm{d}\left(v, t f^{n} v\right) \leq \mathrm{d}(v, t v)+\mathrm{d}\left(t v, t f^{n} v\right) \leq 9+\mathrm{d}\left(v, f^{n} v\right)$. 


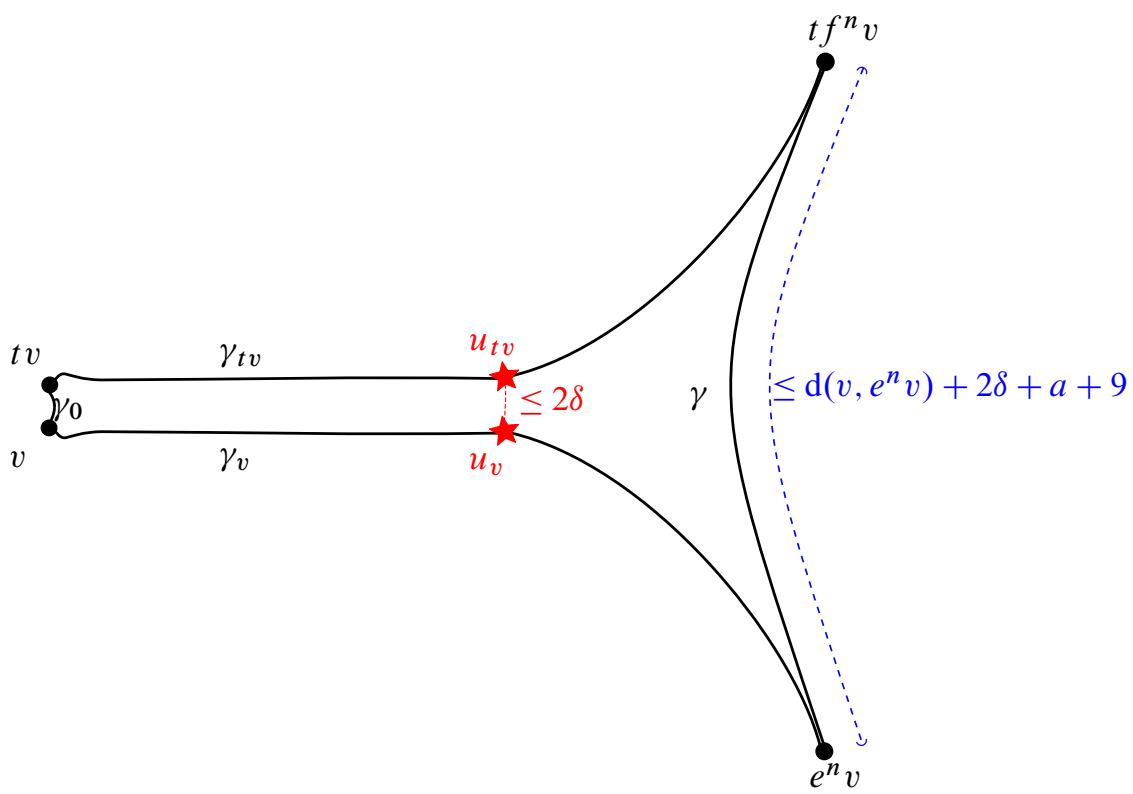

Figure 2: 4-gon in the proof of Theorem 3.

Thus, $\left|\mathrm{d}\left(v, e^{n} v\right)-\mathrm{d}\left(v, t f^{n} v\right)\right| \leq 9$, and, if (4) does not hold, then $\mathrm{d}\left(e^{n} v, t f^{n} v\right) \leq$ $\mathrm{d}\left(v, e^{n} v\right)+2 \delta+a+9$.

Suppose that (4) does not hold for some long enough $n$ that will be specified later. Consider the geodesic 4-gon in $\mathcal{C}_{\mathrm{AL}}(A)$ with geodesics $\gamma_{0}$ from $v$ to $t v, \gamma_{v}$ from $v$ to $e^{n} v$, $\gamma_{t v}$ from $t v$ to $t f^{n} v$ and finally $\gamma$ from $e^{n} v$ to $t f^{n} v$ (see Figure 2). Note that $\gamma_{0}$ has length at most 9 (Corollary 23), and $\gamma$ has length less than $\mathrm{d}\left(v, e^{n} v\right)+2 \delta+a+9$. Let $u_{v}$ be a vertex in $\gamma_{v}$ that is the furthest one away from $v$ with the property of being at distance at most $2 \delta$ of a vertex of $\gamma_{t v}$. Let $u_{t v}$ be the vertex in $\gamma_{t v}$ with $\mathrm{d}\left(u_{v}, u_{t v}\right) \leq 2 \delta$. Note that $\mathrm{d}\left(v, u_{v}\right) \leq 9+\mathrm{d}\left(t v, u_{t v}\right)+2 \delta \leq \mathrm{d}\left(v, u_{v}\right)+18+4 \delta$. Thus $\mathrm{d}\left(v, u_{v}\right)$ and $\mathrm{d}\left(t v, u_{t v}\right)$ only differ by a constant independent of $n$.

We claim that $\mathrm{d}\left(v, u_{v}\right)$ grows linearly with $n$. Indeed, since $u_{v}$ is the vertex in $\gamma_{v}$ furthest away from $v$ with the property of being at distance at most $2 \delta$ of a vertex of $\gamma_{t v}$, there must be a vertex $u$ in $\gamma$ such that $\mathrm{d}\left(u_{v}, u\right) \leq 2 \delta+1$. If $\mathrm{d}\left(u, e^{n} v\right) \leq \frac{1}{2} \mathrm{~d}\left(e^{n} v, t f^{n} v\right)$, then

$$
\mathrm{d}\left(u_{v}, e^{n} v\right) \leq 2 \delta+1+\frac{1}{2} \mathrm{~d}\left(e^{n} v, t f^{n} v\right) \leq 2 \delta+1+\frac{1}{2}\left(\mathrm{~d}\left(v, e^{n} v\right)+2 \delta+9+a\right) .
$$

Since $\mathrm{d}\left(v, e^{n} v\right)=\mathrm{d}\left(v, u_{v}\right)+\mathrm{d}\left(u_{v}, e^{n} v\right)$, we see $\mathrm{d}\left(v, u_{v}\right)$ grow as $\frac{1}{2} \mathrm{~d}\left(v, e^{n} v\right) \sim \frac{1}{4} n$. We similarly derive the same conclusion if $\mathrm{d}\left(u, t f^{n} v\right) \leq \frac{1}{2} \mathrm{~d}\left(e^{n} v, t f^{n} v\right)$, since then 
$\mathrm{d}\left(u_{t v}, t f^{n} v\right) \leq 4 \delta+1+\frac{1}{2} \mathrm{~d}\left(e^{n} v, t f^{n} v\right)$, and $\mathrm{d}\left(v, u_{v}\right)$ and $\mathrm{d}\left(t v, u_{t v}\right)$ only differ by a constant independent of $n$. Therefore, by increasing $n$ we can make $\mathrm{d}\left(v, u_{v}\right)$ as large as needed.

Fix geodesic paths $p_{0}, p_{0}^{-1}$ in $\mathcal{C}_{\mathrm{AL}}(A)$ from $v$ to $g v$ and $v$ to $g^{-1} v$, respectively. Without loss of generality, $p_{0}^{-1}$ is the path $g^{-1} p_{0}$ traversed backwards. Since $|\widetilde{g}|_{\mathcal{S}} \leq 12, p_{0}$ and $p_{0}^{-1}$ have length less than 12 . For $m>0$, we write $p\left(g^{m}\right)$ to denote the path consisting in concatenating $p_{0}, g p_{0}, \ldots, g^{m-1} p_{0}$. Similarly, for $m<0$, we write $p\left(g^{m}\right)$ to denote the path consisting in concatenating $p_{0}^{-1}, g^{-1} p_{0}^{-1}, \ldots, g^{-m+1} p_{0}^{-1}$. As $g$ acts loxodromically, and $\mathrm{d}\left(v, g^{ \pm n} v\right) \geq \frac{1}{2} n$, the paths $p\left(e^{n}\right)$ and $p\left(f^{n}\right)$ are $(\lambda, c)-$ quasigeodesics for $\lambda=24$ and $c=24$. Now, since $\mathcal{C}_{\mathrm{AL}}(A)$ is $\delta$-hyperbolic, there is some constant $\varkappa=\varkappa(\delta, \lambda, c)=\varkappa(60,24,24)$ such that any $(\lambda, c)$-quasigeodesic is in the $x$-neighbourhood of a geodesic path with the same initial and final vertices.

By increasing $n$, we can guarantee that $\gamma_{v}$ and $\gamma_{t} v$ have arbitrarily long initial subpaths that lie in the $2 \delta$-neighbourhood of each other. Since $p\left(e^{n}\right)$ is in the $x$-neighbourhood of $\gamma_{v}$ and $t p\left(f^{n}\right)$ is in the $\varkappa$-neighbourhood of $\gamma_{t v}$, we can take arbitrarily long initial subpaths of $p\left(e^{n}\right)$ and $t p\left(f^{n}\right)$, say $p\left(e^{k}\right)$ and $t p\left(f^{k}\right)$, such that they lie in the $\left(2 x+2 \delta+\ell\left(p_{0}\right)\right)$-neighbourhood of each other. Moreover, it is a standard argument to show that there must be a constant $D=D\left(\lambda, c, \varkappa, \delta, \ell\left(p_{0}\right)\right)$ such that $p\left(e^{k}\right)$ and $t p\left(f^{k}\right)$ synchronously $D$-fellow-travel and, in particular,

$$
\mathrm{d}\left(e^{i} v, t f^{i} v\right) \leq D \quad \text { for } i=0,1, \ldots k .
$$

Note that $D$ is independent of $k$, and $k$ can be made as large as needed.

Let $N=N(2 D)=4(2 D)+319$ and $F=F(2 D)=8(2 D)+638$ as in Theorem 24(iii). Take $n$ large enough so that $k>F+N+1$. By increasing $F$, if necessary, we assume $k=F+N+1$. Let $s=F+1$. We are going to apply the WPD condition on $e^{s} v$ and $e^{N} e^{s} v=e^{k} v$. We are going to act by $t f^{i} t^{-1} e^{-i}$ for $i=1, \ldots, F+1$. First, since $\mathrm{d}\left(t f^{(k-i)} v, e^{(k-i)} v\right) \leq D$ and $\mathrm{d}\left(t f^{(s-i)} v, e^{(s-i)} v\right) \leq D$, we have

$$
\mathrm{d}\left(t f^{k} v, t f^{i} t^{-1} e^{(k-i)} v\right) \leq D \quad \text { and } \quad \mathrm{d}\left(t f^{s} v, t f^{i} t^{-1} e^{(s-i)} v\right) \leq D .
$$

As $\mathrm{d}\left(t f^{k} v, e^{k} v\right) \leq D$, we conclude that

$$
\begin{aligned}
\mathrm{d}\left(\left(t f^{i} t^{-1} e^{-i}\right) e^{k} v, e^{k} v\right) & =\mathrm{d}\left(t f^{i} t^{-1} e^{k-i} v, e^{k} v\right) \\
& \leq \mathrm{d}\left(t f^{i} t^{-1} e^{(k-i)} v, t f^{k} v\right)+\mathrm{d}\left(t f^{k} v, e^{k} v\right) \leq 2 D .
\end{aligned}
$$

An analogous argument, using that $\mathrm{d}\left(t f^{s} v, e^{s} v\right) \leq D$, gives that

$$
\mathrm{d}\left(\left(t f^{i} t^{-1} e^{-i}\right) e^{s} v, e^{s} v\right) \leq 2 D .
$$


By the WPD condition, there are $1 \leq i<j \leq F+1$ such that $t f^{i} t^{-1} e^{-i}=$ $t f^{j} t^{-1} e^{-j}$ and thus, as $e, f \in\left\{g^{-1}, g\right\}, t g^{i-j} t^{-1}=g^{i-j}$ or $t g^{i-j} t^{-1}=g^{j-i}$. Hence $t \in E_{A / Z(A)}(g)$ has the desired contradiction.

Corollary 26 Let $A_{X}$ be a proper standard parabolic subgroup of an irreducible ArtinTits group $A \neq A_{1}, A_{2}, I_{2 m}$ of spherical type. Let $v=\langle\Delta\rangle$ be a vertex in $\mathcal{C}_{\mathrm{AL}}(A)$ and let $g$ be the element in Theorem 24. Denote by $\delta=60$ the hyperbolicity constant of $\mathcal{C}_{\mathrm{AL}}(A)$ and $\mathrm{d}:=\mathrm{d}_{\mathcal{C}_{\mathrm{AL}}}$. Let $a \geq 0$ and $n$ big enough. Then, for all $\epsilon, \eta \in\{-1,1\}$ and for any nontrivial $t, t^{\prime} \in\left(A_{X} \cdot Z(A)\right) / Z(A)$, the following hold:

$$
\begin{aligned}
\mathrm{d}\left(v, g^{2 n} v\right) & \geq \mathrm{d}\left(v, g^{n} v\right)+2 \delta+a, \\
\mathrm{~d}\left(g^{\epsilon n} v, t g^{\eta n} v\right) & \geq \max \left\{\mathrm{d}\left(v, g^{\epsilon n} v\right), \mathrm{d}\left(v, t g^{\eta n} v\right)\right\}+2 \delta+a, \\
\mathrm{~d}\left(v, g^{2 n} v\right) & \geq \max \left\{\mathrm{d}\left(v, \operatorname{tg}^{n} v\right), \mathrm{d}\left(v, g^{n} v\right)\right\}+2 \delta+a, \\
\mathrm{~d}\left(g^{\epsilon n} t^{\prime} v, t g^{\eta n} v\right) & \geq \max \left\{\mathrm{d}\left(v, g^{\epsilon n} t^{\prime} v\right), \mathrm{d}\left(v, \operatorname{tg}^{\eta n} v\right)\right\}+2 \delta+a .
\end{aligned}
$$

Proof Claim (5) follows from the fact that $g$ is loxodromic. Claim (6) was proved in the previous lemma.

Recall that by Corollary $23, \mathrm{~d}(v, t v) \leq 9$ for all $t \in\left(A_{X} \cdot Z(A)\right) / Z(A)$. Let $a \in A / Z(A)$ and $u$ an arbitrary vertex. Observe the following: $\mathrm{d}(a t v, u) \leq \mathrm{d}(a t v, a v)+\mathrm{d}(a v, u)=$ $\mathrm{d}(t v, v)+\mathrm{d}(a v, u) \leq 9+\mathrm{d}(a v, u)$ and, similarly, $\mathrm{d}(a v, u) \leq \mathrm{d}(a v, a t v)+\mathrm{d}(a t v, u) \leq$ $9+\mathrm{d}(a t v, u)$. Therefore,

$$
|\mathrm{d}(a t v, u)-\mathrm{d}(a v, u)| \leq 9 .
$$

Also observe that $\mathrm{d}(v, t a v)=\mathrm{d}\left(t^{-1} v, a v\right) \leq \mathrm{d}\left(t^{-1} v, v\right)+\mathrm{d}(v, a v) \leq 9+\mathrm{d}(v, a v)$, so $\mathrm{d}(v, t a v)-\mathrm{d}(v, a v) \leq 9$. Similarly, $\mathrm{d}(v, a v) \leq \mathrm{d}\left(v, t^{-1} v\right)+\mathrm{d}\left(t^{-1} v, a v\right) \leq 9+\mathrm{d}(v, t a v)$, so $\mathrm{d}(v, a v)-\mathrm{d}(v, t a v) \leq 9$. Therefore,

$$
|\mathrm{d}(v, t a v)-\mathrm{d}(v, a v)| \leq 9 .
$$

For claim (7), we have that $\left|\mathrm{d}\left(v, g^{2 n} v\right)-\mathrm{d}\left(v, \operatorname{tg}^{2 n} v\right)\right| \leq 9$ and $\mathrm{d}\left(v, g^{n} v\right)+2 \delta+a+9 \geq$ $\max \left\{\mathrm{d}\left(v, g^{n} v\right), \mathrm{d}\left(v, g^{n} v\right)\right\}$. Thus (7) follows from the fact that $g$ is loxodromic. Finally, claim (8) follows from the previous lemma and

$$
\left|\mathrm{d}\left(g^{\epsilon n} t^{\prime} v, \operatorname{tg}^{\eta n} v\right)-\mathrm{d}\left(g^{\epsilon n} v, \operatorname{tg}^{\eta n} v\right)\right| \leq 9,
$$

and

$$
\left|\max \left\{\mathrm{d}\left(v, g^{\epsilon n} t^{\prime} v\right), \mathrm{d}\left(v, g^{\eta n} v\right)\right\}-\max \left\{\mathrm{d}\left(v, g^{\epsilon n} v\right), \mathrm{d}\left(v, t g^{\eta n} v\right)\right\}\right| \leq 9 .
$$




\subsection{Proof of Theorem 3}

The strategy to prove Theorem 3 relies on the application of the following lemma.

Lemma 27 [13, Lemma 1.1] Let $\left(x_{i}\right)$ be a sequence of points on a $\delta$-hyperbolic geodesic metric space such that

$$
\mathrm{d}\left(x_{i+2}, x_{i}\right) \geq \max \left\{\mathrm{d}\left(x_{i+2}, x_{i+1}\right), \mathrm{d}\left(x_{i+1}, x_{i}\right)\right\}+2 \delta+a .
$$

Then $\mathrm{d}\left(x_{i}, x_{j}\right) \geq a|j-i|$.

We proceed now to prove that there is a free product complement:

Proof of Theorem 3 Let $v=\langle\Delta\rangle$ be a vertex in $\mathcal{C}_{\mathrm{AL}}(A)$ and let $g$ be the element in Theorem 24. Fix $a>0$ and $n$ big enough that the conclusions of Corollary 26 hold.

We are going to prove that a reduced word $w$ over $\mathcal{A}=\left\langle g^{n}, g^{-n}\right\rangle \cup\left(A_{X} \cdot Z(A)\right) / Z(A)$ represents a nontrivial element of $A / Z(A)$. Indeed, if $w$ is a reduced word over $\mathcal{A}$ then it has the form

$$
w=t_{1} g^{k_{1} n} t_{2} g^{k_{2} n} \cdots t_{s} g^{k_{s} n},
$$

with $k_{i} \in \mathbb{Z} \backslash\{0\}$ for $i<s$ and $k_{s} \in \mathbb{Z}$, and $t_{i} \in\left(A_{X} \cdot Z(A)\right) / Z(A) \backslash\{1\}$ for $i>1$, and $t_{1} \in\left(A_{X} \cdot Z(A)\right) / Z(A)$. If $s=1$, we already know that $w$ cannot represent the identity since $\langle g\rangle$ and $\left(A_{X} \cdot Z(A)\right) / Z(A)$ have trivial intersection. Thus we can assume that $s>1$. If $t_{1}=1$, conjugating $w$ by $g_{1}^{k_{1} n}$ and then performing free reductions, we obtain a shorter word $w^{\prime}=t_{1}^{\prime} g^{k_{1}^{\prime} n} t_{2}^{\prime} g^{k_{2}^{\prime} n} \cdots t_{s^{\prime}}^{\prime} g^{k_{s^{\prime}}^{\prime} n}$ (with respect to the alphabet $\mathcal{A}$ ) with the new $t_{1}^{\prime} \neq 1$. Moreover, $w$ represents the trivial element if and only if $w^{\prime}$ does. So we will assume that $t_{1} \neq 1$. With a similar argument, we can assume that $k_{s} \neq 0$.

Let sgn: $\mathbb{Z} \rightarrow\{-1,0,1\}$ be the sign function. We can consider the orbit of $v$ under prefixes of $w$. Since $t_{1} \neq 1$ and $k_{s} \neq 0$ we can view $w$ as a word over $\left\{g^{n}, g^{-n}, \operatorname{tg}^{n}, \operatorname{tg}^{-n}: t \in A_{X} \cdot Z(A) / Z(A)\right\}$, having the following sequence of vertices $\left\{x_{i}\right\}_{i=0}^{\sum_{i=0}\left|k_{i}\right|}$ in $\mathcal{C}_{\mathrm{AL}}$ :

$$
\begin{gathered}
x_{0}=v, \quad x_{1}=\left(t_{1} g^{\operatorname{sgn}\left(k_{1}\right) n}\right) v, \quad x_{2}=\left(t_{1} g^{\operatorname{sgn}\left(k_{1}\right) 2 n}\right) v, \quad \ldots, \quad x_{\left|k_{1}\right|}=\left(t_{1} g^{k_{1} n}\right) v, \\
x_{\left|k_{1}\right|+1}=\left(t_{1} g^{k_{1} n}\right)\left(t_{2} g^{\operatorname{sgn}\left(k_{2}\right) n}\right) v, \quad x_{\left|k_{1}\right|+2}=\left(t_{1} g^{k_{1} n}\right)\left(t_{2} g^{\operatorname{sgn}\left(k_{2}\right) 2 n}\right) v, \quad \ldots, \\
x_{\left|k_{1}\right|+\left|k_{2}\right|}=\left(t_{1} g^{k_{1} n}\right)\left(t_{2} g^{k_{2} n}\right) v, \quad \ldots, \quad x_{\sum\left|k_{i}\right|}=t_{1} g^{k_{1} n} t_{2} g^{k_{2} n} \cdots t_{s} g^{k_{s} n} v=w v .
\end{gathered}
$$

We want to check that this sequence of vertices satisfies the hypothesis of Lemma 27. We can suppose that $x_{i}=h v$. Then we have several possibilities: 
- Assume that $x_{i+1}=h g^{\epsilon n} v$, with $\epsilon \in\{-1,1\}$. If $x_{i+2}=h g^{2 \epsilon n} v$, use (5) to check the inequality of the hypothesis. If on the other hand $x_{i+2}=h g^{\epsilon n} t_{j} v g^{\eta n}$, with $\eta \in\{-1,1\}$, use (6).

- Assume that $x_{i+1}=h t_{j} g^{\epsilon n} v$. If $x_{i+2}=h t_{j} g^{2 \epsilon n} v$, use (7) to check the desired inequality. If on the other hand $x_{i+2}=h t_{j} g^{\epsilon n} t_{j+1} g^{\eta n} v$ with $\eta \in\{-1,1\}$, use (8).

Then, by Lemma 27, we have that

$$
\mathrm{d}(v, w v)=\mathrm{d}\left(x_{0}, x_{\sum\left|k_{i}\right|}\right) \geq a\left(\sum_{i=1}^{s}\left|k_{i}\right|\right) .
$$

In particular, $\mathrm{d}(v, w v)=0$ if and only if $w=t_{1}$ and hence, since $t_{1} \neq 1, w$ does not represent the trivial element

Hence, we have that $\left(\left\langle A_{X}, g^{n}\right\rangle \cdot Z(A)\right) / Z(A) \leqslant A / Z(A)$ is isomorphic to

$$
\left(\left(A_{X} \cdot Z(A)\right) / Z(A)\right) *\left(\left(\left\langle g^{n}\right\rangle \cdot Z(A)\right) / Z(A)\right) .
$$

Since $Z(A)$ is generated by a power of $\Delta$ and we have that $\langle\Delta\rangle \cap A_{X}=\{1\}=$ $\left\langle g^{n}\right\rangle \cap\langle\Delta\rangle$, we get that $\left(\left\langle A_{X}, g^{n}\right\rangle \cdot Z(A)\right) / Z(A) \leqslant A / Z(A)$ is isomorphic to $A_{X} *\left\langle g^{n}\right\rangle$. Fix a preimage $\widetilde{g}$ of $g$ in $A$. We know that $\widetilde{g}$ can be taken to be positive with length at most 12. Finally, notice that $A_{X} *\left\langle\widetilde{g}^{n}\right\rangle$ maps onto $\left\langle A_{X}, \widetilde{g}^{n}\right\rangle \leqslant A$, which maps onto $\left(\left\langle A_{X}, g^{n}\right\rangle \cdot Z(A)\right) / Z(A) \cong A_{X} *\left\langle g^{n}\right\rangle$. Therefore, $\left\langle A_{X}, \widetilde{g}^{n}\right\rangle \cong A_{X} *\left\langle\widetilde{g}^{n}\right\rangle$ and $g_{*}=\widetilde{g}^{n}$.

Remark 28 By Corollary 23, $N_{A}\left(A_{X}\right)$ (the normalizer of $A_{X}$ in $A$ ) acts elliptically with a diameter bounded by 9 . Note that $\Delta^{2} \in N_{A}\left(A_{X}\right)$ and therefore $\left\langle N_{Z}(A), \widetilde{g}^{n}\right\rangle$ cannot be a free product since $\Delta^{2}$ commutes with $\tilde{g}$ and hence $\left\langle N_{Z}(A), \widetilde{g}^{n}\right\rangle$ has nontrivial centre. However, if one takes $H \leqslant N_{A}\left(A_{X}\right)$ such that $H \cap Z(A)=\{1\}$ then our proof shows that $\left\langle H, \widetilde{g}^{n}\right\rangle \cong H *\left\langle\widetilde{g}^{n}\right\rangle$.

\section{Exponential growth rate}

In this last section we prove the Corollary 4 about exponential growth rate of parabolic subgroups with respect to the Garside generating set.

The coproduct in the category of monoids is constructed in the same way as in groups. If $A$ and $B$ are two monoids, their coproduct is denoted by $A * B$. Its elements are 
reduced words in $A \cup B$ and its operation is the concatenation (followed by reduction). If $M$ is a monoid and $T$ is some subset, we write $\langle T\rangle^{+}$for the submonoid generated by $T .{ }^{1}$ To prove Corollary 4 we need the following lemma, which is a standard application of generating functions (see [18, VI.A, Proposition 4]), modified to give some weight to a free generator.

Lemma 29 Let $G$ be a group, $\mathcal{S}$ be a finite generating set and $M$ be a submonoid. Let $1 \leq \alpha \leq \omega(M, \mathcal{S})$. Suppose that $g \in G$ satisfies that $|g|_{\mathcal{S}} \leq k$ and $\langle M, g\rangle^{+} \cong M *\langle g\rangle^{+}$. Also let $\gamma$ be the positive root of $1-\alpha x-x^{k}$. Then $1 / \gamma>\alpha$ is a lower bound for $\omega\left(\langle M, g\rangle^{+}, \mathcal{S}\right)$.

In particular, if $M$ is a subgroup, since $\langle M, g\rangle$ contains $\langle M, g\rangle^{+}$we have that $1 / \gamma>\alpha$ is a lower bound for $\omega(\langle M, g\rangle, \mathcal{S})$.

Proof Let $\beta_{M}(n)=\#\left\{h \in M:|h|_{\mathcal{S}} \leq n\right\}$. Since $\beta_{M}$ is submultiplicative, Fekete's lemma implies that

$$
\alpha \leq \omega(M, \mathcal{S})=\lim _{n \rightarrow \infty} \sqrt[n]{\beta_{M}(n)}=\inf _{n \geq 1} \sqrt[n]{\beta_{M}(n)}
$$

exists and therefore $\alpha^{n} \leq \beta_{M}(n)$ for all $n \geq 1$.

Now, an element of $t \in\langle M, g\rangle^{+}$has a unique expression written in the form

$$
m_{1} g^{n_{1}} m_{2} g^{n_{2}} m_{3} \cdots m_{\ell} g^{n_{\ell}},
$$

where $m_{i} \in M$ with $m_{i} \neq 1$ for $i>1$, and $n_{i} \in \mathbb{Z}_{\geq 0}$ with $n_{i} \neq 0$ for $i \leq \ell$. The $\mathcal{S}$-length of the element in (9) is bounded above by

$$
\|t\|:=\sum_{i=1}^{\ell}\left|m_{i}\right|_{\mathcal{S}}+|g|_{\mathcal{S}} \cdot\left(\sum_{i=1}^{\ell} n_{i}\right) .
$$

Notice that $|t|_{\mathcal{S}} \leq\|t\|$. Thus

$$
\beta_{\langle M, g\rangle^{+}}(n) \geq \beta_{\langle M, g\rangle^{+}}^{\|\cdot\|}(n):=\#\left\{t \in\langle M, g\rangle^{+}:\|t\| \leq n\right\} .
$$

We are going to estimate the growth rate of $\beta_{\langle M, g\rangle^{+}}^{\|\cdot\|}(n)$. For that, for $s \in \mathbb{N}$ let $L(s) \subseteq\langle M, g\rangle^{+}$denote the subset of elements of $\langle M, g\rangle^{+}$that when written in the form (9) give $\ell=s$, that is,

$$
\begin{aligned}
L(s)=\left\{m_{1} g^{n_{1}} \cdots m_{s} g^{n_{s}} \in\langle M, g\rangle^{+}: m_{i} \in M, n_{i} \in \mathbb{Z}_{\geq 0} \text { for all } i,\right. \\
\left.m_{i} \neq 1 \text { for } i>1, n_{i} \neq 0 \text { for } i<s\right\} .
\end{aligned}
$$

\footnotetext{
${ }^{1}$ The notation $\langle Y\rangle^{+}$usually is reserved for the subsemigroup generated by $Y$. Note that the subsemigroup generated by a set $T$ and the submonoid generated by $T$ just differ by one element and thus the growth rates are equal.
} 
Since $\bigsqcup_{s \geq 1} L(s)=\langle M, g\rangle^{+}$, letting $\beta_{L(s)}^{\|\cdot\|}(n)$ denote $\#\{t \in L(s):\|t\| \leq n\}$ we have that

$$
\beta_{\langle M, g\rangle^{+}}^{\|\cdot\|}(n)=\sum_{s \geq 0} \beta_{L(s)}^{\|\cdot\|}(n)
$$

For a function $\beta: \mathbb{N} \rightarrow \mathbb{N}$, we denote by $\mathcal{G}_{\beta}(x)$ the growth series $\sum_{n \geq 0} \beta(n) x^{n}$. Also let

$$
\mathcal{G}_{1}(x):=\sum_{n \geq 0} \alpha^{n} x^{n}=\frac{1}{1-\alpha x} \quad \text { and } \quad \mathcal{G}_{2}(x):=\sum_{n \geq 1}\left(x^{|g|_{\mathcal{S}}}\right)^{n}=\frac{1}{1-x^{|g|_{\mathcal{S}}}} .
$$

Since $\beta_{M}(n) \geq \alpha^{n}$, we have that $\mathcal{G}_{\beta_{M}}(x) \geq \mathcal{G}_{1}(x)$. Also observe $\beta_{\langle g\rangle}(n) \geq 1$ and thus $\mathcal{G}_{\beta_{\langle g\rangle}+}(x) \geq \mathcal{G}_{2}(x)$. Then

$$
\begin{aligned}
\mathcal{G}_{\beta_{L(s)}^{\|\cdot\|}}(x) & =\mathcal{G}_{\beta_{M}}(x)\left(\left(\mathcal{G}_{\beta_{\langle g\rangle}+}(x)-1\right)\left(\mathcal{G}_{\beta_{M}}(x)-1\right)\right)^{s-1} \mathcal{G}_{\beta_{\langle g\rangle}+}(x) \\
& \geq \mathcal{G}_{1}(x)\left(\left(\mathcal{G}_{2}(x)-1\right)\left(\mathcal{G}_{1}(x)-1\right)\right)^{s-1} \mathcal{G}_{2}(x)
\end{aligned}
$$

for $s \geq 1$. Using (10) and (11) and the previous inequality, we get that

$$
\begin{aligned}
\mathcal{G}_{\beta_{\langle M, g\rangle}+} & \geq \sum_{s \geq 1} \mathcal{G}_{\beta_{L(s)}^{\|\cdot\|}}(x) \\
& \geq \sum_{s \geq 1} \mathcal{G}_{1}(x)\left(\left(\mathcal{G}_{2}(x)-1\right)\left(\mathcal{G}_{1}(x)-1\right)\right)^{s-1} \mathcal{G}_{2}(x) \\
& =\mathcal{G}_{1}(x) \mathcal{G}_{2}(x) \sum_{s \geq 1}\left(\left(\mathcal{G}_{2}(x)-1\right)\left(\mathcal{G}_{1}(x)-1\right)\right)^{s-1} \\
& =\mathcal{G}_{1}(x) \mathcal{G}_{2}(x) \frac{1}{1-\left(\mathcal{G}_{2}(x)-1\right)\left(\mathcal{G}_{1}(x)-1\right)} \\
& =\frac{1}{1-\alpha x-x^{|g|_{S}}} .
\end{aligned}
$$

Therefore, the exponential growth rate of $\beta_{\langle M, g\rangle^{+}}(n)$ is bounded below by the inverse of the convergence radius of the growth series of $\left(1-\alpha x-x^{|g|_{S}}\right)^{-1}$ which is the positive root $\gamma_{0}$ of $1-\alpha x-x^{|g|_{S}}$. It is easy to see that $1 / \gamma_{0}$ is strictly greater than $\alpha$ and, moreover, if $|g|_{\mathcal{S}} \leq k$, then the positive root $\gamma$ of $1-\alpha x-x^{k}$ is bigger than $\gamma_{0}$ (and thus $1 / \gamma<1 / \gamma_{0}$ is a lower bound for the growth rate).

To finally prove Corollary 4, we need one more result. This is a result that is well known for Artin-Tits groups of spherical type (see an explanation in [9, Section 3]), which has been generalized for Garside groups in [17, Theorem 1.13]: 
Lemma 30 Let $A$ be an Artin-Tits group of spherical type, $A_{X}$ be a parabolic subgroup and $\mathcal{S}$ the set of Garside generators of $A$. Then $\mathcal{S} \cap A_{X}$ is the set of Garside generators of $A_{X}$ and the embedding of $A_{X}$ into $A$ with respect to the Garside generators is isometric, that is, for every $g \in A_{X}$ the length of $g$ with respect to $\mathcal{S}^{ \pm}$ and $\mathcal{A} \cap \mathcal{S}^{ \pm 1}$ is the same.

Proof of Corollary 4 Let $A$ be an Artin-Tits group of spherical type and $\mathcal{S}$ be its set of Garside generators of $A$. Let $A_{X}$ be a proper parabolic subgroup. Suppose first that $A=A_{1}$. Then $A$ is cyclic and the only proper parabolic subgroup is trivial. It is easy to see that $\omega\left(A_{X}, \mathcal{S}^{ \pm 1}\right)=\omega\left(A, \mathcal{S}^{ \pm 1}\right)=1$. Now suppose that $A$ is equal to either $A_{2}$ or $I_{2 m}$. Both of these groups (and their monoids) have exponential growth since they contain nonabelian free semigroups. Therefore $\omega\left(A, \mathcal{S}^{ \pm 1}\right)>1$. However, the only proper parabolic subgroups are trivial or cyclic and thus $\omega\left(A_{X}, \mathcal{S}^{ \pm 1}\right)<\omega\left(A, \mathcal{S}^{ \pm 1}\right)$.

Henceforth, we assume that $A \neq A_{1}, A_{2}, I_{2 m}$. By Theorem 3, there is a constant $K$ and an element $g \in A$ satisfying $|g|_{\mathcal{S}} \leq K$ such that $\left\langle A_{X}, g\right\rangle \cong A_{X} *\langle g\rangle$. By Lemma 29, we have that $\omega\left(A_{X}, \mathcal{S}^{ \pm 1}\right)<\omega\left(\left\langle A_{X}, g\right\rangle, \mathcal{S}^{ \pm 1}\right)$. By definition, we also have $\omega\left(\left\langle A_{X}, g\right\rangle, \mathcal{S}^{ \pm 1}\right) \leq \omega\left(A, \mathcal{S}^{ \pm 1}\right)$. Notice that, since $\left\langle A_{X}, g\right\rangle \cong A_{X} *\langle g\rangle$, the submonoid $\left\langle A_{X}^{+}, g\right\rangle^{+}$is isomorphic to the coproduct of monoids $A_{X}^{+} *\langle g\rangle^{+}$and similarly $\omega\left(A_{X}^{+}, \mathcal{S}\right)<\omega\left(A^{+}, \mathcal{S}\right)$. This proves the first claim of the corollary.

Let us show that the sequence $\left\{\omega\left(A_{n}, \mathcal{S}_{A_{n}}^{ \pm 1}\right)\right\}_{n=1}$ goes to infinity. The proofs for the other five claims are analogous. Consider each $A_{i}$ sitting inside $A_{i+1}$ as a standard parabolic subgroup $A_{1} \leqslant A_{2} \leqslant A_{3} \leqslant \cdots$. Observe that by Theorem 24 there are $g_{i} \in A_{i}$ for $i=1,2,3, \ldots$ satisfying $\left|g_{i}\right|_{\mathcal{S}_{A_{i}}} \leq K$ and such that $\left\langle A_{i-1}, g_{i}, g_{i+1}, \ldots, g_{i+s}\right\rangle \leqslant A_{i+s}$ is isomorphic to $A_{i-1} *\left\langle g_{i}\right\rangle *\left\langle g_{i+1}\right\rangle * \cdots *\left\langle g_{i+s}\right\rangle$. Thus we can use Lemma 29 inductively to get a lower bound for $\omega\left(A_{i+s}, \mathcal{S}_{A_{i+s}}^{ \pm 1}\right)$. Notice that, by Lemma 30, we have $\omega\left(A_{i}, \mathcal{S}_{A_{i}}^{ \pm 1}\right)=\omega\left(A_{i}, \mathcal{S}_{A_{n}}^{ \pm 1}\right)$ for every $n \geq i$. Let $\alpha_{1}=\omega\left(A_{1}, \mathcal{S}_{A_{1}}^{ \pm 1}\right)=1$. For $i \geq 1$, let $\gamma_{i}$ be the root of $1-\alpha_{i} x-x^{K}$ and let $\alpha_{i+1}=1 / \gamma_{i}$. By the previous discussion and Lemma 29, $\alpha_{i} \leq \omega\left(A_{i}, \mathcal{S}_{A_{i}}^{ \pm 1}\right)$. So it is enough to show that $\left\{\alpha_{i}\right\}$ goes to infinity. Note that, also by Lemma 29, $\alpha_{i}<\alpha_{i+1}$ for all $i \in \mathbb{N}$. So the sequence $\left\{\alpha_{i}\right\}$ is increasing and either converges or goes to infinity. If it converges to some value, say $\eta$, then $1 / \eta$ must be a root of $0=1-\eta x-x^{K}$. But this means that $0=1 / \eta^{K}$. Therefore, $\left\{\alpha_{i}\right\}_{i=1}^{\infty}$ diverges and then $\left\{\omega\left(A_{n}, \mathcal{S}_{A_{n}}^{ \pm 1}\right)\right\}_{n=1}$ goes to infinity.

Acknowledgments Antolín acknowledges partial support from the Spanish Government through grants number MTM2014-53810-C2-01, MTM2017-82690-P and through 
the "Severo Ochoa Programme for Centres of Excellence in R\&D" (SEV-2015-0554). Cumplido acknowledges the support of the projects MTM2016-76453-C2-1-P (financed by the Spanish Government and FEDER) and FQM-2018 (financed by Junta de Andalucía). She also thanks the University of Burgundy for financing her with a one-year postdoc contract. We thank Bert Wiest for suggesting we prove Theorem 2 and José M Conde-Alonso for useful discussions. We also thank Juan González-Meneses for reading the first part of this article and helping to improve it.

We are very grateful to the referee of this paper for his/her careful reading and thoughtful report.

\section{References}

[1] E Artin, Theory of braids, Ann. of Math. 48 (1947) 101-126 MR Zbl

[2] M Bestvina, K Fujiwara, Bounded cohomology of subgroups of mapping class groups, Geom. Topol. 6 (2002) 69-89 MR Zbl

[3] E Brieskorn, K Saito, Artin-Gruppen und Coxeter-Gruppen, Invent. Math. 17 (1972) 245-271 MR Zbl

[4] M Calvez, B Wiest, Acylindrical hyperbolicity and Artin-Tits groups of spherical type, Geom. Dedicata 191 (2017) 199-215 MR Zbl

[5] M Calvez, B Wiest, Curve graphs and Garside groups, Geom. Dedicata 188 (2017) 195-213 MR Zbl

[6] M Calvez, B Wiest, Hyperbolic structures for Artin-Tits groups of spherical type, preprint (2019) arXiv To appear in Contemp. Math.

[7] HS M Coxeter, The complete enumeration of finite groups of the form $r_{i}^{2}=\left(r_{i} r_{j}\right)^{k_{i j}}=$ 1, J. Lond. Math. Soc. 10 (1935) 21-25 Zbl

[8] M Cumplido, On the minimal positive standardizer of a parabolic subgroup of an Artin-Tits group, J. Algebraic Combin. 49 (2019) 337-359 MR Zbl

[9] M Cumplido, V Gebhardt, J González-Meneses, B Wiest, On parabolic subgroups of Artin-Tits groups of spherical type, Adv. Math. 352 (2019) 572-610 MR Zbl

[10] F Dahmani, V Guirardel, D Osin, Hyperbolically embedded subgroups and rotating families in groups acting on hyperbolic spaces, Mem. Amer. Math. Soc. 1156, Amer. Math. Soc., Providence, RI (2017) MR Zbl

[11] P Dehornoy, L Paris, Gaussian groups and Garside groups, two generalisations of Artin groups, Proc. Lond. Math. Soc. 79 (1999) 569-604 MR Zbl

[12] P Deligne, Les immeubles des groupes de tresses généralisés, Invent. Math. 17 (1972) 273-302 MR Zbl 
[13] T Delzant, Sous-groupes à deux générateurs des groupes hyperboliques, from "Group theory from a geometrical viewpoint" (É Ghys, A Haefliger, A Verjovsky, editors), World Sci., River Edge, NJ (1991) 177-189 MR Zbl

[14] R Flores, J González-Meneses, On the growth of Artin-Tits monoids and the partial theta function, preprint (2018) arXiv

[15] E Godelle, Normalisateurs et centralisateurs des sous-groupes paraboliques dans les groupes d'Artin-Tits, $\mathrm{PhD}$ thesis, Université de Picardie Jules Verne (2001)

[16] E Godelle, Normalisateur et groupe d'Artin de type sphérique, J. Algebra 269 (2003) 263-274 MR Zbl

[17] E Godelle, Parabolic subgroups of Garside groups, J. Algebra 317 (2007) 1-16 MR $\mathrm{Zbl}$

[18] P de la Harpe, Topics in geometric group theory, Univ. Chicago Press (2000) MR Zbl

[19] H van der Lek, The homotopy type of complex hyperplane complements, $\mathrm{PhD}$ thesis, Katholieke Universiteit Nijmegen (1983)

[20] L Paris, Parabolic subgroups of Artin groups, J. Algebra 196 (1997) 369-399 MR Zbl

[21] L Paris, Artin monoids inject in their groups, Comment. Math. Helv. 77 (2002) 609-637 MR Zbl

YA: Departamento de Matemáticas, Universidad Autónoma de Madrid Instituto de Ciencias Matemáticas

Madrid, Spain

Current address: Algebra Geometría y Topología, Universidad Complutense de Madrid Madrid, Spain

MC: IMB, UMR 5584, CNRS, Université Bourgogne Franche-Comté Dijon, France

Current address: Departamento de Álgebra, Universidad de Sevilla Sevilla, Spain yago.anpi@gmail.com, mariacumplidocabello@gmail.com

Received: 23 September 2019 Revised: 10 July 2020 